\title{
Stabilization of Antiferromagnetic Order in FeO Nanolayers
}

\author{
S. Couet, ${ }^{1,2, *}$ K. Schlage, ${ }^{1}$ R. Rüffer, ${ }^{3}$ S. Stankov,${ }^{3 \dagger}$ Th. Diederich, ${ }^{1}$ B. Laenens,${ }^{2}$ and R. Röhlsberger ${ }^{1}$ \\ ${ }^{1}$ Deutsches Elektronen Synchrotron (DESY), Notkestraße 85, 22603 Hamburg, Germany \\ ${ }^{2}$ K.U.Leuven, Instituut voor Kern- en Stralingsfysica \& INPAC, Celestijnenlaan 200D, B-3001 Leuven, Belgium \\ ${ }^{3}$ European Synchrotron Radiation Facility (ESRF), BP 220, 38043 Grenoble cedex, France
}

(Received 18 June 2009; published 26 August 2009)

\begin{abstract}
We have studied the evolution of the magnetic state of a nanometer thick antiferromagnetic (AFM) FeO layer during its formation using nuclear resonant scattering of synchrotron radiation. In contact to ferromagnetic $\mathrm{Fe}$, the $\mathrm{FeO}$ layer does not show magnetic order at room temperature (RT). Once embedded between two Fe layers, magnetic coupling to the adjacent ferromagnets leads to a drastic increase of the Néel temperature far above RT, while the blocking temperature remains below $30 \mathrm{~K}$. The presented results evidence the role that the ferromagnetic surrounding plays in modifying the magnetic state of ultrathin AFM layers.
\end{abstract}

DOI: 10.1103/PhysRevLett.103.097201

Magnetic data storage technology is reaching the bottom of the nanoscale. The stabilization of magnetic order in low-dimensional structures becomes a key issue. Below a critical thickness, ferromagnets undergo a superparamagnetic relaxation where spins are thermally excited, and the system does not show magnetic hysteresis anymore. Exchange bias bilayer systems composed of a ferromagnet (FM) and an antiferromagnet (AFM) have been recently reported to substantially reduce the critical thickness under which the ferromagnet undergoes superparamagnetic relaxation $[1,2]$. Layered FM/AFM systems, where an AFM layer (with high anisotropy) is used to pin the magnetization of the FM, are used to create reference magnetic layers in devices. Below a certain temperature (the blocking temperature $T_{B}$ ) the spins in the antiferromagnet are frozen and exchange coupling at the FM/AFM interface leads to a shift of the hysteresis loop and an increase of the coercivity, this phenomenon being known as the exchange bias effect $[3,4]$.

In the ultrathin film limit, the thermal stability of these FM/AFM bilayers is strongly modified [5,6]. In exchange bias systems, one usually distinguishes the Néel (ordering) temperature $T_{N}$ of the antiferromagnet and the blocking temperature $T_{B}$ below which exchange bias occurs [7]. For thick AFM layers $(>50 \mathrm{~nm}), T_{N}$ and $T_{B}$ are usually found to be equivalent. Field cooling the system below $T_{N}$ directly leads to the freezing of spins (and the appearance of exchange bias). Early studies showed that $T_{B}$ was usually decreasing with decreasing thickness of the AFM layer, and it was assumed that $T_{N}$ was decreasing accordingly due to a finite size effect [5]. Recently, Vallejo-Fernandez et al. found out that the evolution of the exchange bias field $H_{e}$ and $T_{B}$ for polycrystalline films was in fact linked to the average grain volume of the AFM [6], which typically decreases with the layer thickness. This results in a decreased stability against thermal excitation and a decrease of $T_{B}$. It should be noted that there is still an underlying physics problem as data on epitaxially grown AFM/FM
PACS numbers: 75.70.Cn, 75.25.+z, 75.75.+a, 76.80.+y

bilayers also show a reduction of $T_{B}$ with a decreasing AFM layer thickness [8,9]; this is still the subject of different theoretical considerations $[5,10]$. The evolution of $T_{N}$ relative to $T_{B}$ was investigated by Van der Zaag et al. on $\mathrm{Fe}_{3} \mathrm{O}_{4} / \mathrm{CoO} / \mathrm{Fe}_{3} \mathrm{O}_{4}$ multilayers [9]. They found that $T_{N}$ and $T_{B}$ differ in the ultrathin film limit i.e., $T_{N}$ is increasing and $T_{B}$ is decreasing with decreasing AFM layer thickness. The increase of $T_{N}$ was interpreted using a mean field theory, which describes the influence of the surrounding spins as a mean magnetic field acting on the spins in the FM and AFM layers [9].

In this Letter, we present an in situ study of the formation of an ultrathin antiferromagnetic $\mathrm{FeO}$ layer on $\mathrm{Fe}$ using nuclear resonant scattering (NRS) of synchrotron radiation. The isotope sensitivity of the technique is used here to study exclusively the $\mathrm{FeO}$ part of the system via introduction of isotopic probe layers. The sensitivity to the hyperfine interaction of the ${ }^{57} \mathrm{Fe}$ nuclei [11] allows one to follow the evolution of the chemical and magnetic environment of the ${ }^{57} \mathrm{Fe}$ atoms during the growth. We observe that the magnetically disordered $\mathrm{FeO}$ layer undergoes a magnetic transition only once the Fe layer deposited on its top is sufficiently thick to sustain long-range ferromagnetism. Temperature dependent measurements show a drastic increase of $T_{N}$ and the occurrence of exchange bias at low temperature, as predicted in Ref. [9]. These findings demonstrate how ultrathin antiferromagnets can be stabilized by embedding them between thin ferromagnets.

The in situ experiment was carried out in a dedicated ultrahigh vacuum (UHV) chamber [12] at the nuclear resonance beam line of the European Synchrotron Radiation Facility ESRF (Grenoble, France) ID18 [13]. First, a $2 \mathrm{~nm}$ thick ${ }^{56} \mathrm{Fe}$ layer was deposited on a W(110) single crystal with a $0.5 \mathrm{~nm}{ }^{57} \mathrm{Fe}$ layer on its top. This isotopic substitution does not modify the chemical and magnetic structure of the system but ensures that only the ${ }^{57} \mathrm{Fe}$ part of the sample is probed via NRS. At this stage the nuclear time spectrum (the time dependent deexcitation of 
the ${ }^{57} \mathrm{Fe}$ nucleus after pulsed excitation using synchrotron radiation [11]) shows a single frequency beating associated with a hyperfine field $B_{\text {hf }}$ of $32.6 \mathrm{~T}$ [see Fig. 1(a)], typical for bcc Fe at room temperature (RT). The layer was subsequently oxidized by admission of molecular oxygen at a pressure of $1 \times 10^{-5}$ mbar for $500 \mathrm{~s}$. A drastic change is seen [Fig. 1(b)] in the time spectrum and the hyperfine field distribution (HFD). Two components can be identified: one with a large $B_{\mathrm{hf}}$ centered around $29 \mathrm{~T}(35 \%)$ and several low $B_{\mathrm{hf}}$ distributions accounting for nonmagnetic states. This collection of sites is consistent with the disordered oxide state reported in Ref. [14] for saturated native iron oxide layers, where it is also shown that this oxidation procedure leads to a disordered (eventually amorphous) crystalline structure. The oxide layer is then reduced to the desired $\mathrm{FeO}$ structure by deposition of $0.8 \mathrm{~nm}$ of ${ }^{56} \mathrm{Fe}$. A $1.6 \mathrm{~nm} \mathrm{FeO}$ layer enriched in ${ }^{57} \mathrm{Fe}$ is formed. It should be stressed that the study reported in [14] showed that the oxide is completely reduced to $\mathrm{FeO}$ after deposition of the first $\mathrm{Fe}$ monolayer. The additional $\mathrm{Fe}$ remains on top in a pure metallic state. The corresponding time spectrum [Fig. 1(c)] is fitted using a main $B_{\mathrm{hf}}$ component ( $\left.\sim 90 \%\right)$ centered at $5 \mathrm{~T}$, attributed to nonmagnetic $\mathrm{FeO}$. So one can expect that $T_{N}$ does not significantly increase or is lower than the bulk value (bulk $T_{N}=198 \mathrm{~K}$ ), although the layer

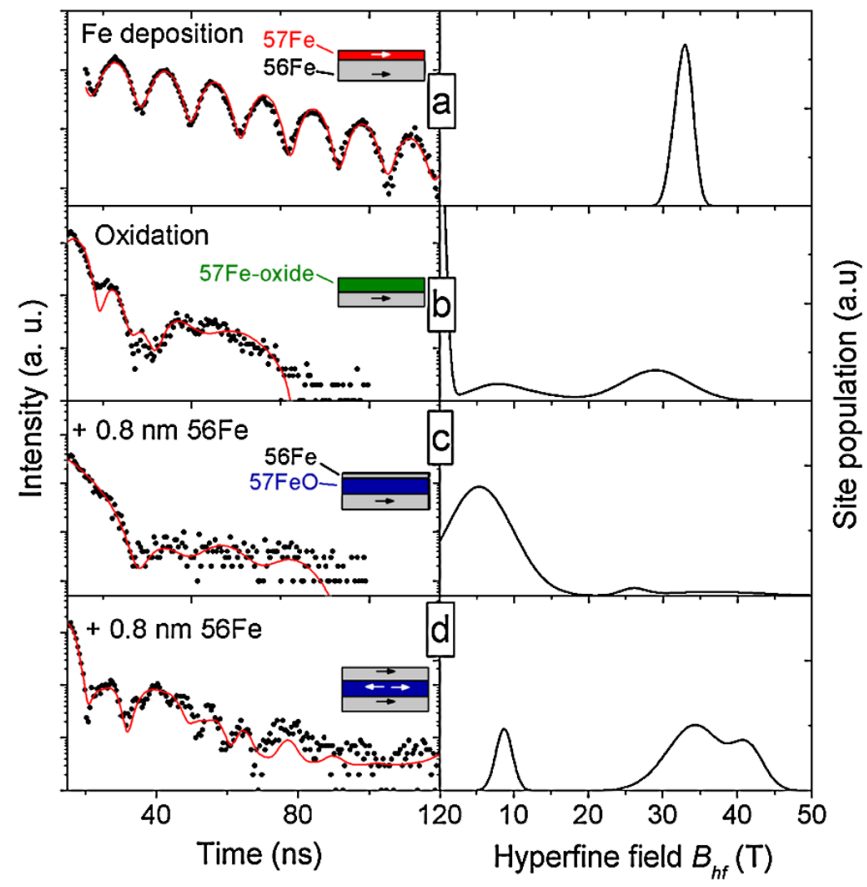

FIG. 1 (color online). Nuclear time spectra (left panels) and corresponding hyperfine field distributions (right panels) recorded during the growth of an $\mathrm{Fe} /$ native-oxide/Fe trilayer at room temperature. The solid lines on the time spectra are fits. The $1.6 \mathrm{~nm}$ buried $\mathrm{FeO}$ layer appears to be magnetically ordered only when the top Fe layer is thick enough to develop long-range magnetic order. is in contact at one of its interfaces with a ferromagnetic Fe layer.

The deposition of a second $0.8 \mathrm{~nm}$ thick ${ }^{56} \mathrm{Fe}$ layer (which brings a nominal ${ }^{56} \mathrm{Fe}$ thickness of $1.6 \mathrm{~nm}$ on top of the $\mathrm{FeO}$ layer) drastically changes the picture [see Fig. 1(d)] when high frequency beatings, associated with large values of $B_{\mathrm{hf}}$, appear in the time spectrum. It is known that this further deposition of Fe does not modify the chemical structure of the $\mathrm{FeO}$ nanolayer (which is already in the most reduced state [14]). The observed changes are attributed to a modification of the magnetic interaction that the $\mathrm{FeO}$ layer experiences. In fact, one can expect that this second deposition goes along with the transition to long-range magnetic order in the top Fe layer. One distinguishes mainly two sites in the HFD, which are attributed to $\mathrm{Fe}$ atoms either fully coordinated to oxygen atoms and interfacial atoms with slightly different environment. The appearance of magnetic ordering in the $\mathrm{FeO}$ layer indicates that $T_{N}$ is effectively increased only when the AFM layer is embedded between FM layers. These findings complement very well the stabilization mechanism proposed by Van der Zaag et al. [9]: The Néel temperature of ultrathin AFM can be effectively increased in the case of buried layers while this seems not to be the case for a free AFM layer.

We investigated ex situ the temperature dependence of the magnetic state of the embedded $\mathrm{FeO}$ layer on a similar system which exhibits the layer sequence Si-substrate/ $\mathrm{Ta} /{ }^{56} \mathrm{Fe}(2 \mathrm{~nm}) /{ }^{57} \mathrm{FeO}(1.5 \mathrm{~nm}) /{ }^{56} \mathrm{Fe}(2 \mathrm{~nm}) /$ Ta. NRS time spectra were recorded in the temperature range $10 \mathrm{~K}<\mathrm{T}<400 \mathrm{~K}$ to follow the evolution of the corresponding HFD. Two representative spectra recorded at 10

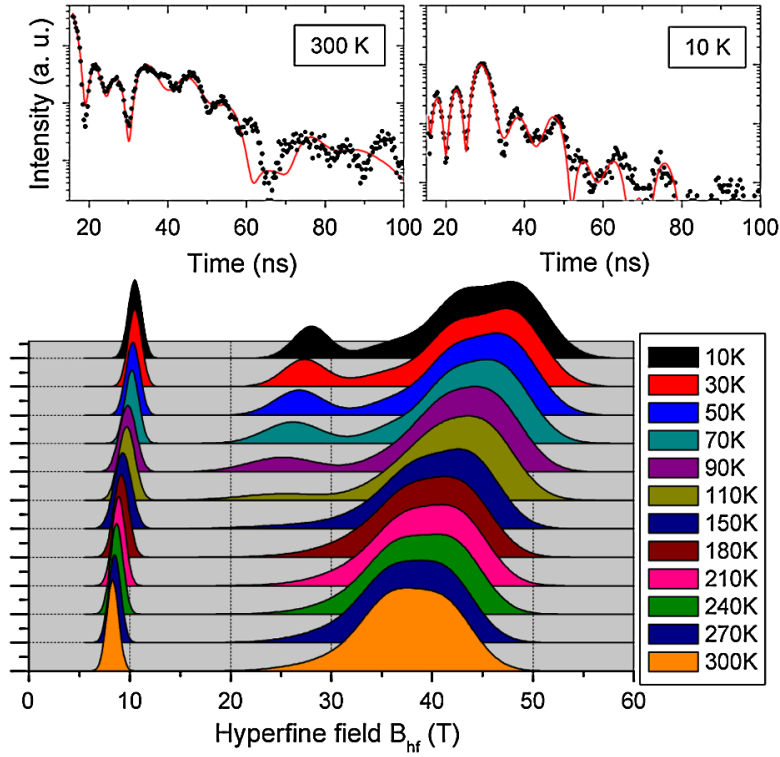

FIG. 2 (color online). Upper panels: Nuclear time spectra recorded on a ${ }^{56} \mathrm{Fe} /{ }^{57} \mathrm{FeO}(1.6 \mathrm{~nm}) /{ }^{56} \mathrm{Fe}$ trilayer at $300 \mathrm{~K}$ and at $10 \mathrm{~K}$. The solid lines are fits. Lower panel: Hyperfine field distributions extracted from the fits of the time spectra. 
and $300 \mathrm{~K}$ and the extracted HFD are displayed in Fig. 2. At $300 \mathrm{~K}$, the HFD is similar to the one recorded in situ [Fig. 1(d)], proving that the magnetic state of the $\mathrm{FeO}$ layer is essentially the same. Taking the relative disorder of this FeO layer (created by oxidation) [14], the low hyperfine field contribution centered around 8-10 $\mathrm{T}$ is expected to originate from $\mathrm{Fe}$ atoms at grain boundaries or in interstitial positions.

The magnitude of $B_{\mathrm{hf}}$ is in first approximation proportional to the local magnetic moment of the atoms. The evolution of the mean value of the large $B_{\mathrm{hf}}(\mathrm{FeO})$ component with temperature is a good indicator of the stability of the antiferromagnetic state. It is seen in Fig. 3 that $B_{\mathrm{hf}}(\mathrm{FeO})$ is decreasing only slowly up to $400 \mathrm{~K}$. Above this temperature, thermal diffusion started to irreversibly change the structure of the sample. A fit of the curve using a law of the form [15]

$$
B_{\mathrm{hf}} \propto m_{\mathrm{AFM}} \propto\left(T_{N}-T\right)^{1 / 3}
$$

where $m_{\mathrm{AFM}}$ is the magnetization of the $\mathrm{Fe}$ in the $\mathrm{FeO}$ layer, yields a $T_{N}$ of $800 \pm 30 \mathrm{~K}$, a value much larger than the bulk value of $198 \mathrm{~K}$. This large increase of $T_{N}$ is in accordance with the prediction of the mean field theory used to describe the evolution of the magnetic order in ultrathin FM/AFM superlattices $[9,16,17]$. In the case of a $\mathrm{FM} / \mathrm{AFM}$ system, the Curie temperature $T_{C}$ of the FM is only weakly dependent on the relative thickness of the FM and AFM layers [17]. In contrast, the $T_{N}$ of the AFM layer is gradually approaching $T_{C}$ when its thickness is reduced. This mean field approach usually considers the coupling experienced by the spins in the center of each layer with its nearest neighbors under periodic boundary conditions which are compatible with a superlattice structure. As we obviously see here, this type of mechanism allows us to understand the behavior of the buried $\mathrm{FeO}$ layer, but cannot be used to describe the $\mathrm{Fe} / \mathrm{FeO}$ bilayer system, where the $\mathrm{FeO}$ exhibits one free interface. In that case, the antiferromagnet seems to behave essentially as an isolated AFM layer, i.e., one can expect that $T_{N}$ is not modified [9] or is eventually decreased due to the increasing relative number of interfacial (loosely coupled) spins [5].

The second phenomenon associated with the ultrathin nature of the AFM is the decrease of $T_{B}$ with decreasing

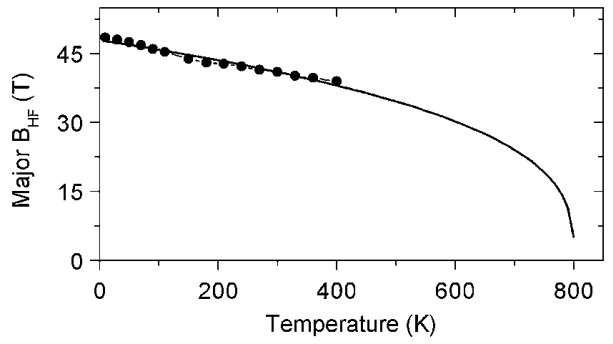

FIG. 3. Temperature dependence of the mean value of the large $B_{\mathrm{hf}}$ component displayed in Fig. 2. The solid line is a fit using a $\left(T_{N}-T\right)^{1 / 3}$ law, which yield a Néel temperature of $800 \pm 30 \mathrm{~K}$. thickness. For temperatures $T$ between $T_{B}$ and $T_{N}$ the spins in the AFM are free to rotate their magnetization vectors with respect to the ferromagnet. As a time-resolved technique, NRS constitutes a very sensitive probe of relaxation dynamics in magnetic systems [18]. The scattering process extends over a time scale that is determined by the lifetime $\tau$ of the excited nuclear level ( $141 \mathrm{~ns}$ in the case of ${ }^{57} \mathrm{Fe}$ ). If the relaxation time of the magnetic fluctuations is significantly shorter than $\tau$, the magnetic signal averages out and the sample appears to be nonmagnetic. With decreasing temperature the fluctuation time eventually becomes larger than the scattering time, i.e., the measurement yields a snapshot of the sample where the magnetic order seems to be frozen in. Figure 2 displays evidence for such a transition in our sample: at about $110 \mathrm{~K}$ a broad hyperfine field component appears around $25 \mathrm{~T}$ that shifts towards $28 \mathrm{~T}$ with decreasing temperature. It is most likely related to the freezing of spins at the interface or within the AFM. In the time spectra it manifests as an additional modulation that leads to a significant intensity enhancement between 20 and $30 \mathrm{~ns}$.

For comparison, Fig. 4(a) shows temperature dependent magnetization curves recorded by vibrating-sample magnetometry (VSM) after the same sample was cooled in an external field $B_{\text {ext }}$ of $200 \mathrm{mT}$. At $10 \mathrm{~K}$, the most prominent features of the exchange bias are seen, namely, an increase of the coercive field $H_{c}$ and a shift of the hysteresis loop. At $50 \mathrm{~K}$, there is no exchange bias field while the coercivity remains larger than at RT. The evolution of $H_{c}$ and $H_{e}$ with temperature displayed in Fig. 4(b) clearly shows that the coercive field $H_{c}$ starts to increase at a much higher tem-
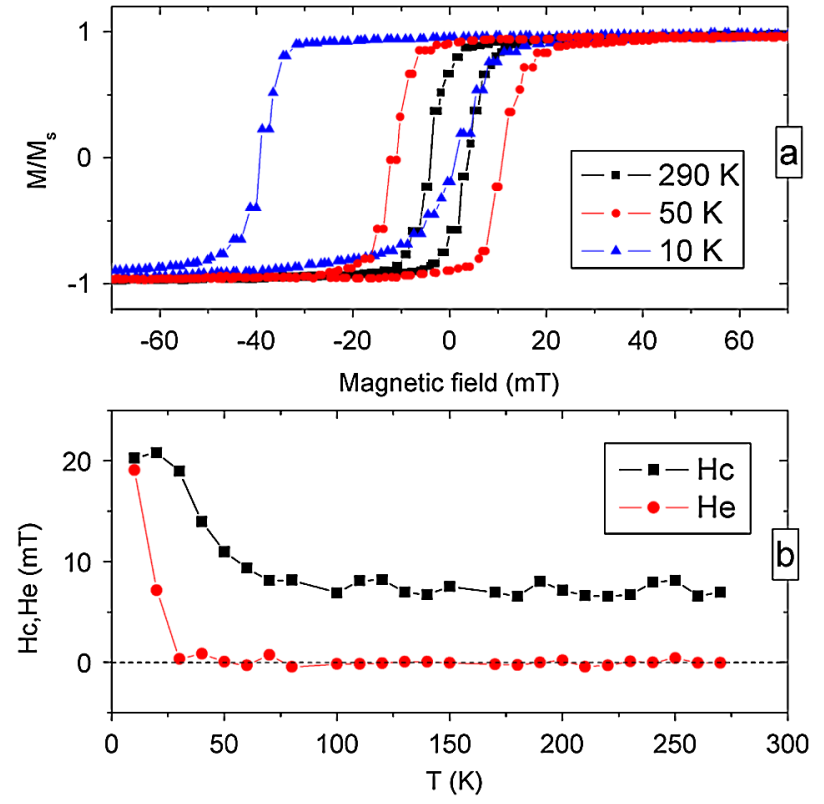

FIG. 4 (color online). (a) Selected hysteresis curves recorded by VSM on a $\mathrm{Fe} / \mathrm{FeO}(1.6 \mathrm{~nm}) / \mathrm{Fe}$ trilayer. (b) Temperature dependence of the coercive field $H_{c}$ and exchange bias field $H_{e}$ extracted from the magnetization curves. 
perature than the exchange bias field $H_{e}$, an effect reported to occur in ultrathin AFM layers [19]. Fernandez-Outon et al. have shown that spontaneous magnetization of interfacial spins in exchange bias systems can lead to a drastic increase of $H_{e}$ [20], an effect which eventually depends on the amount of structural disorder in the system. In fact, the increase of $H_{e}$ that we observe below $30 \mathrm{~K}$ is consistent with the evolution of the $28 \mathrm{~T}$ component in Fig. 2. The rapid broadening of this distribution for temperatures above $30 \mathrm{~K}$ is a sign that the corresponding atoms start to loose their fixed magnetic orientation. The fact that the distribution does not evolve much between 10 and $30 \mathrm{~K}$ indicates that we have reached the nominal value of the hyperfine field, i.e., that spins are not fluctuating anymore, leading to the rapidly rising exchange bias field. We conclude that the freezing of spins plays a crucial role for the appearance of exchange bias in this ultrathin disordered $\mathrm{FeO}$ layers grown by oxidation.

In conclusion, we have observed in situ the evolution of the magnetic state of a $1.6 \mathrm{~nm}$ thick antiferromagnetic $\mathrm{FeO}$ layer. We see that the presence of only one FM/AFM interface is not sufficient to stabilize the AFM ordering at RT while the deposition of a second ferromagnetic layer leads to a strong increase of $T_{N}$. This latter system then behaves as predicted by the mean field theory. The most important conclusion is that a free (ultrathin) AFM layer (even supported by a ferromagnet) does not experience an increase of its Néel temperature. While this cannot be directly generalized to the case of metallic AFM (such as those used in devices), it shows the importance the ferromagnetic surrounding plays in stabilizing AFM layers. Evidence for a similar effect in metallic systems would therefore bring insight into the mechanism that leads to the effect we observed in this oxide based antiferromagnet. We also see that the magnetization of interfacial spins plays a major role for the observation of the exchange bias effect in this ultrathin $\mathrm{Fe} / \mathrm{FeO}$ systems. Additional studies on similar systems such as $\mathrm{CoO} / \mathrm{Co}$ or $\mathrm{NiO} / \mathrm{Ni}$ would allow us to eventually generalize this concept. Finally, these direct experimental observations show the importance of understanding the mutual influence of thin ferromagnets on thin antiferromagnets for the design of possible future magnetic systems incorporating ultrathin antiferromagnetic layers.

The authors gratefully acknowledge the NRS group of the ESRF as well as J. Korecki, M. Zajac, and J. Meersschaut for their precious help during the in situ experiment. This work was supported by the European Commission under FP6 Contract No. NMP4-CT-2003001516 DYNASYNC. *sebastien.couet@fys.kuleuven.be

${ }^{\dagger}$ Now at the Institute for Synchrotron Radiation, Forschungszentrum Karlsruhe.

[1] V. Skumryev, S. Stoyanov, Y. Zhang, G. Hadjipanayis, D. Givord, and J. Nogués, Nature (London) 423, 850 (2003).

[2] E. Fullerton, D. Margulies, M. Schabes, M. Carey, B. Gurney, A. Moser, M. Best, G. Zeltzer, K. Rubin, H. Rosen, and M. Doerner, Appl. Phys. Lett. 77, 3806 (2000).

[3] J. Nogués and I. K. Schuller, J. Magn. Magn. Mater. 192, 203 (1999).

[4] A.E. Berkowitz and K. Takano, J. Magn. Magn. Mater. 200, 552 (1999).

[5] Y. J. Tang, D. J. Smith, B. L. Zink, F. Hellman, and A. E. Berkowitz, Phys. Rev. B 67, 054408 (2003).

[6] G. Vallejo-Fernandez, L. E. Fernandez-Outon, and K. O'Grady, J. Phys. D 41, 112001 (2008).

[7] A. N. Dobrynin and R. Prozorov, J. Appl. Phys. 102, 043902 (2007).

[8] R. Jungblut, R. Coehoorn, M. T. Johnson, C. Sauer, P. J. van der Zaag, A. R. Ball, T. G. S. M. Rijks, J. Destegge, and A. Reinders, J. Magn. Magn. Mater. 148, 300 (1995).

[9] P. J. van der Zaag, Y. Ijiri, J. A. Borchers, L. F. Feiner, R. M. Wolf, J.M. Gaines, R.W. Erwin, and M. A. Verheijen, Phys. Rev. Lett. 84, 6102 (2000).

[10] S. S. P. Parkin and V. S. Speriosu, in Magnetic Properties of Low-Dimensional Systems II, edited by L. M. Falicov, F. Mejiia-Lira, and J.L. Moran-Lopez (Springer-Verlag, Heidelberg, 1990), Vol. 50, p. 110

[11] R. Röhlsberger, Nuclear Condensed Matter Physics Using Synchrotron Radiation, Springer Tracts in Modern Physics Vol. 208 (Springer-Verlag, Berlin, 2004).

[12] S. Stankov, R. Rüffer, M. Sladecek, M. Rennhofer, B. Sepiol, G. Vogl, N. Spiridis, T. Slezak, and J. Korecki, Rev. Sci. Instrum. 79, 045108 (2008).

[13] R. Rüffer and A. I. Chumakov, Hyperfine Interact. 97/98, 589 (1996).

[14] S. Couet, K. Schlage, K. Saksl, and R. Röhlsberger, Phys. Rev. Lett. 101, 056101 (2008).

[15] The temperature dependence of the magnetization, and especially the $1 / 3$ exponent used here, is typical for spin systems described by the Heisenberg model.

[16] R. W. Wang and D.L. Mills, Phys. Rev. B 46, 11681 (1992).

[17] J. A. Borchers, R. W. Erwin, S. D. Berry, D. M. Lind, J.F. Ankner, E. Lochner, K. A. Shaw, and D. Hilton, Phys. Rev. B 51, 8276 (1995).

[18] O. Leupold and H. Winkler, Hyperfine Interact. 123/124, 571 (1999).

[19] X. Y. Lang, W. T. Zheng, and Q. Jiang, Nanotechnology 18, 155701 (2007).

[20] L. E. Fernandez-Outon, G. Vallejo-Fernandez, S. Manzoor, B. Hillebrands, and K. O'Grady, J. Appl. Phys. 104, 093907 (2008). 